\title{
Research of Film Uniformity on Vacuum Coating by Magnetron Sputtering with Multi-Sites
}

\author{
Ying Huang ${ }^{1,}$, Shitie Gao ${ }^{1, b}$, Meng Liu ${ }^{1, c}$ \\ ${ }^{1}$ School of Mechanical Engineering \& Automation, Northeastern University, Shenyang 110819, PR \\ China \\ aYinghuang@me.neu.edu.cn, bgaoshitie@163.com, calvinnana@163.com
}

Keywords: Magnetron sputtering, Thickness uniformity, Eccentricity, Target-substrate distance

\begin{abstract}
A new type of multi-site magnetron sputtering system has been researched, which has more than one workbench. Based on the operating principle of the magnetron sputtering system which with a circular plane target, a mathematical model has been developed to simulate and discuss the influencing factors on the film thickness uniformity. The results showed that when the substrates were rotating axially and eccentrically, the film thickness distribution were affected by both the target-substrate distance and the eccentricity. If the eccentricity was constant, the film thickness would become thinner when the target-substrate distance increased, and the thickness uniformity tended to be improved. If the target-substrate distance was constant, the thickness uniformity would become better when the eccentricity increased. Moreover, if the substrate rotated axially and revolved around the target simultaneously, the thickness uniformity would become better when velocity ratio of axial rotation to revolution increased. And the effect of the thickness uniformity became small gradually if the ratio increases to a certain degree. In addition, increasing the etching area properly not only could conduct the film distribution to better, and improve the substrate film thickness uniformity also. Finally, taking a series of experiments, and analyzing the experimental data, the conclusion of the study results in the paper had been verified.
\end{abstract}

\section{The new type system of multi-position magnetron sputtering}

The multi-position magnetron sputtering system which illustrated in Fig.1 could realize rotation and revolution of the work piece.

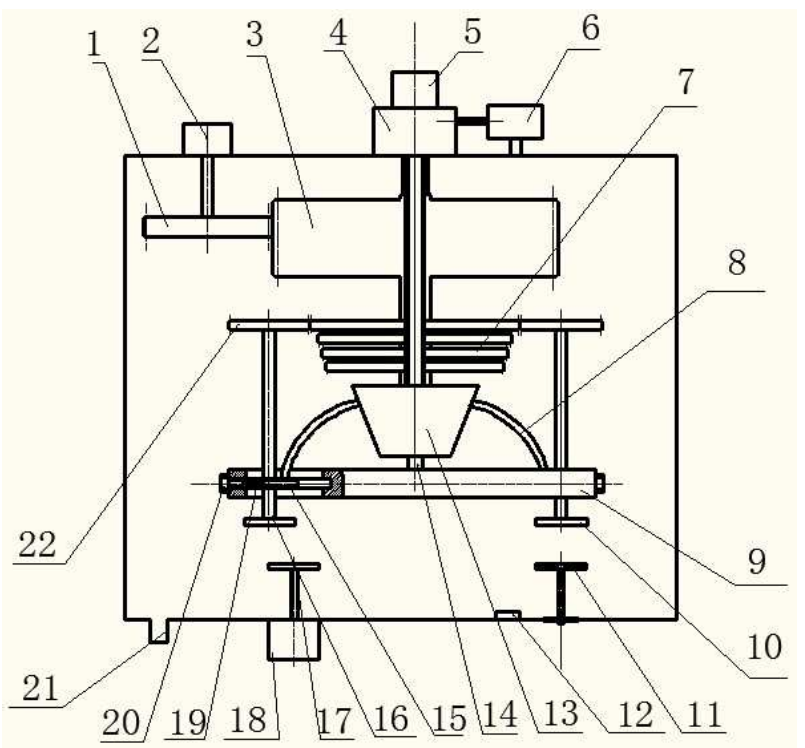

1-motor gear 2-rotation motor 3-middle gear 4-decelerator 5-Eccentricity mobilize Box 6-revolution motor 7-gear unit 8-contact rod 9 -revolution tray 10-workpiece pallet 11-heater

12-aeration hole of target-substrate distance 13-Tapered mobilization 14-axis of revolution 15-slider \& slide 16-axis of rotation 17-magnetron sputtering target 18 -control box 19-spring 20 -bolt 21-extractor vent 22-rotation gear 23-sleeve

Fig. 1. Multi-position diagram of magnetron sputtering system 
The target-substrate distance, eccentricity and the rotating speed are also can be controlled. (1) For the workbench frame, there is no interference between perpetual and rotation movement. Speed of revolution motor and rotation motor can be regulated independently. According to the actual requirements, the rotation and revolution speed ratio can be set automatically to change the point locus. (2) Via the four sliders inside four slides, there are deviations between the center of the substrate and that of the target realizing adjustment of eccentricity. (3) Using the adjustment device that under the target to adjust the vertical position of target and the adjustment between the target and substrate.

\section{Film thickness distribution model}

Physical distribution model. As illustrated in Fig.2, the eccentricity of the target center axis and the rotation axis of substrate as $e$, the vertical distance as $H$, the distance between surface element $\mathrm{d} A$ on the target and the axis of the target as $r$, the distance between surface element $\mathrm{d} B$ on the substrate and the axis of the target as $R$, the center distance between $\mathrm{d} A$ and $\mathrm{d} B$ as $L$, sputtering angle as $\beta$, the angle between the normal of $\mathrm{d} B$ and the line to the center of $\mathrm{d} A$ as $\varphi$, polar angle of $\mathrm{d} A$ as $\theta$. In order not to result in serious errors, the paper made the following assumption:(1) Ion incident angle is zero; (2) Magnetron sputtering rate of the system is proportional to the horizontal component of the magnetron magnetic field in the target surface [2]; (3) Sputtering rate is $p$, the angel of the atom that is sputtering out comply with the cosine distribution, conveyed by $\cos (\beta), \beta$ means the emergence angle; (4) Due to the flight, assuming the emergent particle is scattered. Its deposition rate on the substrate is inversely proportional to the path length $L$.

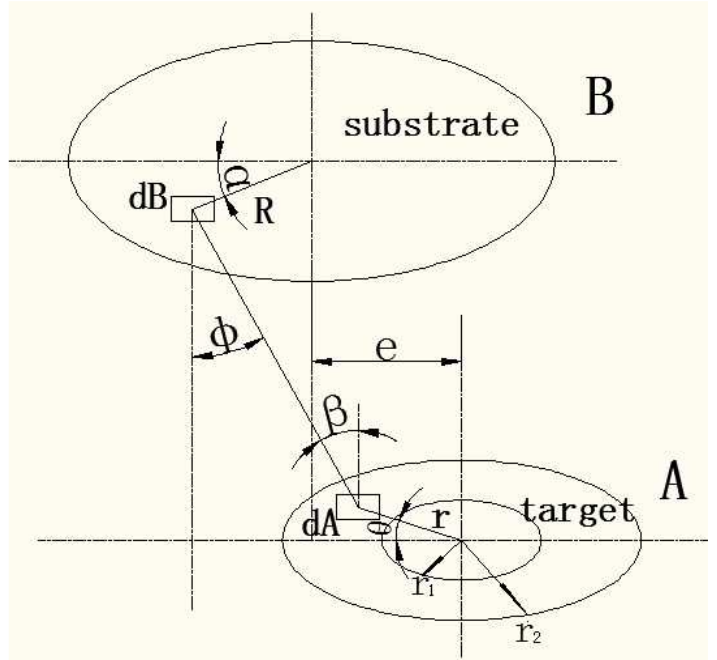

Fig. 2. Schematic diagram of planar magnetron sputtering system with plane target

The mathematical model of film thickness distribution. According to the condition (3), the particle number that from any surface element $\mathrm{d} A$ on the target to the surface element $\mathrm{d} B$ on the substrate is:

$$
\mathrm{d} N=P \cdot \cos (\beta) \cdot \mathrm{d} A \cdot \mathrm{d} \omega / \pi .
$$

where $\beta$ is the angel between the normal of $\mathrm{d} A$ and the attachment from $\mathrm{d} A$ to $\mathrm{d} B, \omega$ is the solid angle, then $d \omega=d B \cdot \cos \varphi / L^{2}, \varphi$ is the angel between the normal of $\mathrm{d} B$ and the attachment between $\mathrm{d} A$ and $\mathrm{d} B$. Due to the parallel arrangement of the sputtering target and the substrate, there is $\beta=\varphi$.

According to the condition (4), the particle number that from any surface element $\mathrm{d} A$ on the target to the surface element $\mathrm{d} B$ on the substrate is:

$\mathrm{d} N_{0}=P \cdot \cos (\beta) \cdot \cos (\phi) \cdot \mathrm{d} A \cdot \mathrm{d} \omega / \pi \mathrm{L}^{2}$.

Assuming the density of target materials is $\rho$, film thickness is $T$, then

$\mathrm{d} N_{0}=\rho \cdot \mathrm{d} T \cdot \mathrm{d} B$. 
So the film thickness at the point $B$ on the substrate is

$$
T=\int P \cdot \cos ^{2}(\beta) \mathrm{d} A / \pi \rho L^{2}
$$

$\mathrm{d} A=r d r d \theta, \cos (\beta)=H / L, H$ is the target-substrate distance, $R$ is the distance between the point $\mathrm{B}$ and the substrate center, $r$ is the distance between the point $\mathrm{A}$ and the target center.

Assuming inside radius and outer radius in the etching area are $r_{1}$ and $r_{2}$, the film thickness at point $\mathrm{A}$ is

$$
T=\frac{P \cdot H^{2}}{\pi \rho} \int_{0}^{2 \pi} \int_{0}^{2 \pi} \int_{r_{1}}^{r_{2}} \frac{r}{L^{4}} \mathrm{~d} r \mathrm{~d} \theta \mathrm{d} \alpha .
$$

The value of $\mathrm{P} / \rho$ is a constant 1 . According to the geometry relationship of Fig.2, then

$$
L^{2}=H^{2}+e^{2}+R^{2}+r^{2}-2 e \cdot r \cdot \cos (\theta)+2 e \cdot R \cdot \cos (\alpha)-2 R \cdot r \cdot \cos (\theta) \cdot \cos (\alpha)-2 R \cdot e \cdot \sin (\theta) \cdot \sin (\alpha) \text {. }
$$

Therefore Eq. 5 can be simplified for

$$
T=\frac{H^{2}}{\pi} \int_{0}^{2 \pi} \int_{0}^{2 \pi} \int_{r_{1}}^{r_{2}} \frac{r}{L^{4}} \mathrm{~d} r \mathrm{~d} \theta \mathrm{d} \alpha .
$$

When the movement of substrate compounds rotation and revolution, establish the coordinate system as Fig. 3. $R^{2}=(x-\mathrm{a})^{2}+y^{2}, x$ and $y$ are separately for the coordinate representation of the points on substrate. $a$ is the distance between rotation center and revolution center of the substrate.

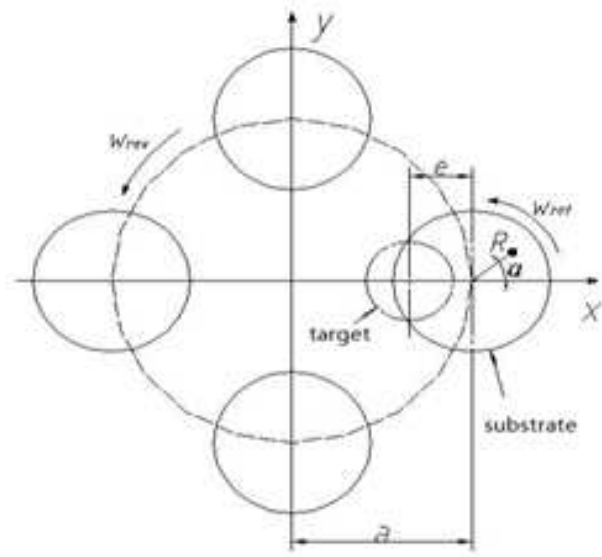

Fig. 3. Position of target relative to substrate during axial rotation and revolution

In polar coordinate, arbitrary point on the substrate such as $\mathrm{C}(R, \alpha)$ comply with Kepler parameter equation.

$$
\left\{\begin{array}{l}
x=R \cdot \cos \left(\omega_{r o t} t+\alpha\right)+a \cdot \cos \left(\omega_{r e v} t\right)=\zeta(t) \\
y=R \cdot \sin \left(\omega_{r o t} t+\alpha\right)+a \cdot \sin \left(\omega_{\text {rev }} t\right)=\psi(t)
\end{array} .\right.
$$

where $\omega_{\text {rot }}$ is the angular velocity of the substrate rotation, $\omega_{\text {rev }}$ is the angular velocity of the substrate frame revolution. $R$ is rotation radius of point $\mathrm{C}$ and $\mathrm{t}$ is the time function. On line integral of the trajectory to point $\mathrm{C}$, then get the film thickness of this point in t time:

$$
T=\int_{L} T_{m}(x, y) d s=\int_{0}^{\tau} \mathrm{d}(\zeta(t), \psi(t)) \cdot \sqrt{\zeta^{2}(t)+\psi^{2}(t)} \mathrm{d} t .
$$

In order to measure the film thickness uniformity, introducing the following statistic: average film thickness value $T$, relative ratio of film thickness $T_{\mathrm{r}}$, mean-absolute deviation $\Delta_{\text {mean }}$, mean-relative deviation $\delta_{\text {mean }}$. 


\section{Analysis and discussions of the film uniformity by magnetron sputtering with multi-workbench}

Pre-set size of the target is $75 \mathrm{~mm}$, the inside radius and outer radius in the etching area are $r_{1}$ for $5 \mathrm{~mm}$ and $r_{2}$ for $30 \mathrm{~mm}$. Substrate size is $100 \mathrm{~mm}$. To use MATLAB investigating the influence of the film thickness uniformity which from the target-substrate distance, eccentricity, angular velocity ratio of rotation to revolution and etching width.

Influence of the target-substrate distance. To calculate the film thickness in different targetsubstrate distance when the eccentricity is $25 \mathrm{~mm}$, etching width is $5-30 \mathrm{~mm}$, then fitting film thickness distribution to find out the radial film thickness.

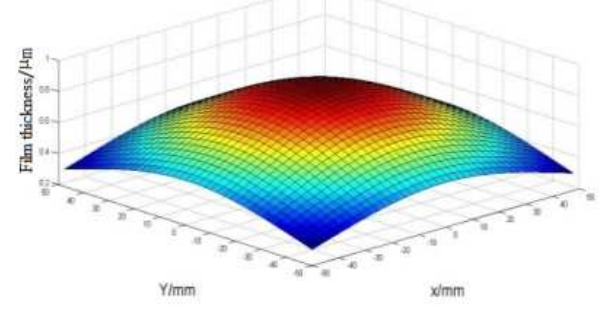

a) $H=70 \mathrm{~mm}$

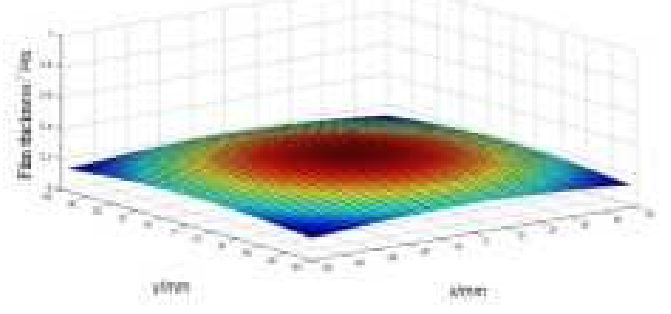

b) $H=90 \mathrm{~mm}$

Fig. 4. Film thickness distribution of different target-substrate distance $H$

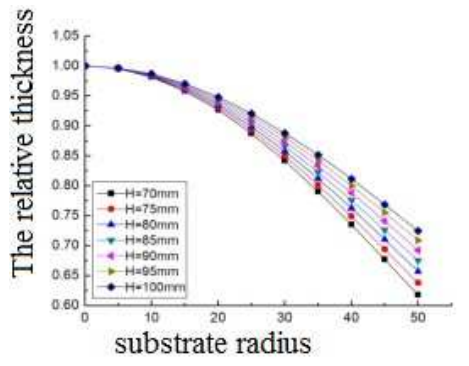

Fig. 5. The relative thickness from radial direction of the substrate

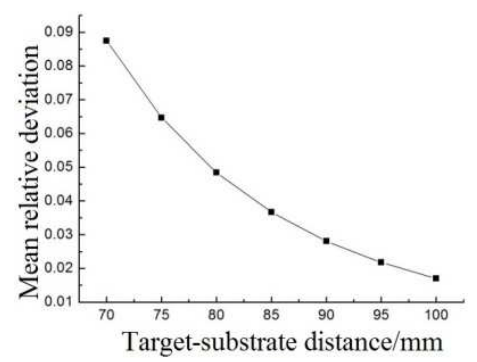

Fig. 6. Relationship between mean relative deviation of film thickness and target-substrate distance

In Fig. 4(a), when the target-substrate distance is $70 \mathrm{~mm}$, the film thickness in center and that at the edges differ obviously. When the target-substrate distance adds to $90 \mathrm{~mm}$, relative thickness decreases (as shown in Fig. 4(b)), that means the dropping deposition rate. Meanwhile, the relative thickness approximate to 1 along with the increasing target-substrate distance (as shown in Fig.5). Preliminary showed that when the substrate in-situ coating with a fixed eccentricity, the film thickness uniformity getting better with the increasing target-substrate distance. As shown in Fig.6, the thickness uniformity is improving.

Influence of eccentricity. To calculate the film thickness in different eccentricity when the target-substrate distance is $70 \mathrm{~mm}$, etching width is $5-30 \mathrm{~mm}$, then fitting film thickness distribution. To find out the radial film thickness and the mean relative deviation that could show the relationship between the film thickness distribution and the eccentricity.

In Fig.7 and Fig.8, with the increasing eccentricity, film thickness deceasing gradually by convex shape tending to gently and becoming sunken finally. So the relative thickness from radial direction of the substrate to be less than 1 then to be close to 1 and more than 1 in the end. As shown in Fig.9, when eccentricity is $0 \mathrm{~mm}$, the mean relative deviation of film thickness is $12.69 \%$, when eccentricity is $60 \mathrm{~mm}$, the mean relative deviation of film thickness decreased to $0.32 \%$ to achieving the optimal. With the increasing eccentricity, film thickness becomes poor gradually. 


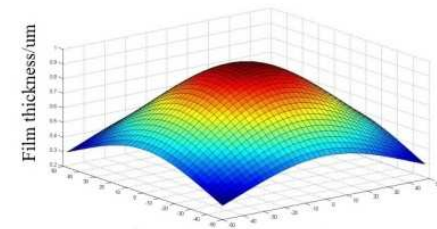

(a) $\mathrm{e}=5 \mathrm{~mm}$

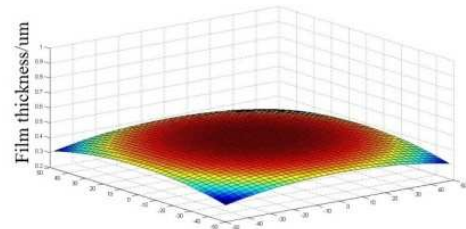

(b) $\mathrm{e}=55 \mathrm{~mm}$

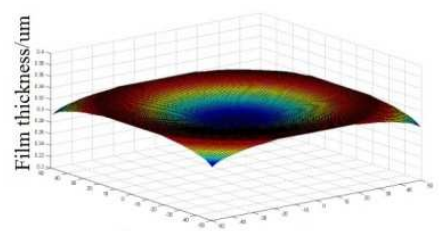

(c) $\mathrm{e}=70 \mathrm{~mm}$

Fig. 7. Film thickness distribution as the function of eccentricity

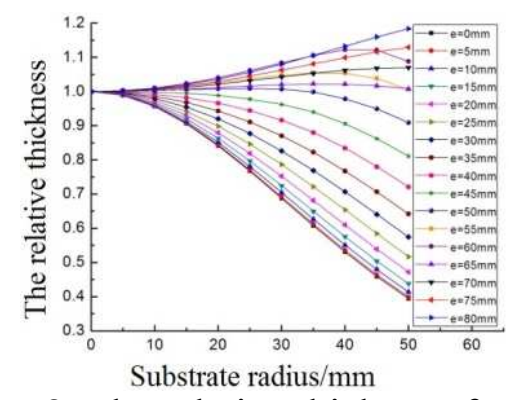

Fig. 8. The relative thickness from radial direction of the substrate

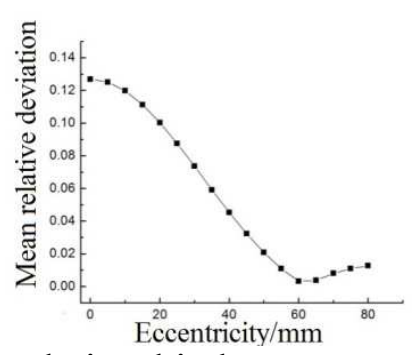

Fig. 9. Relationship between mean relative deviation of film thickness and eccentricity

Influence of the rotation speed and revolution speed. When the ratio is fixed the trajectories of a point are irrelevant to the specific value. When the period of revolution is far less than the sputtering time, the film thickness distribution is certain as long as the relative velocity.

Fig.10 shows the locus of points on substrate at different velocity ratio. When the rotation velocity is low, the locus is sparse. In Fig.10 (b) and Fig.10(c), with the increasing rotation velocity, the locus becomes intensive. So the film thickness uniformity will be improved effectively. For planets rotation form of the substrate frame, the analysis of arbitrary locus on clamps shows that to avoid the rounded ratio to avoid the periodically uneven $[5,6]$. Overall consideration, instead of increasing the velocity ratio, we can just choose the proper motor and velocity, and in this way, not only can it guarantee the well film thickness, but also can reduce energy consumption and save cost.

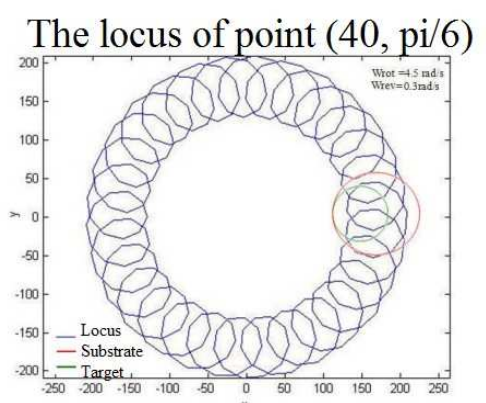

a) $k=15$

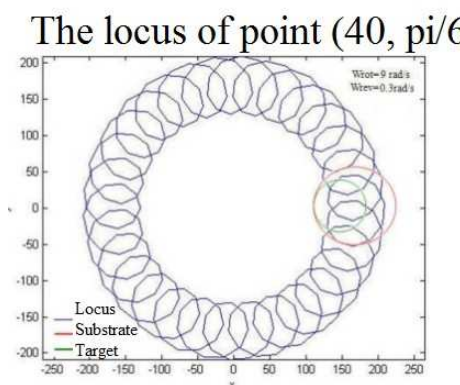

b) $k=30$

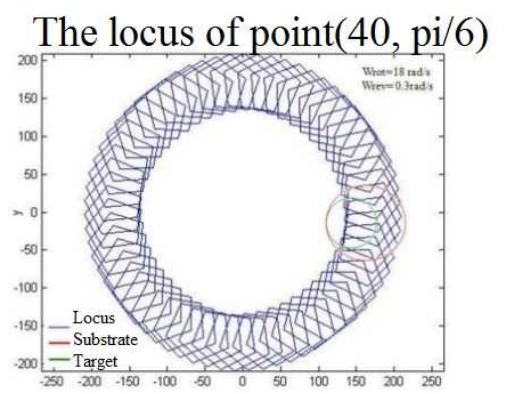

c) $k=60$

Fig. 10. Locus of points on substrate at different velocity ratio $k$

\section{Experiment}

The turntable of frame for this experiment can revolves from $0^{\circ}-360^{\circ}$. The four substrates are uniform in $\varphi 260$ on the pitch circle and the largest size of substrate is $\varphi 50$. The substrate rotating speed is $5-50 \mathrm{r} / \mathrm{min}$. The three targets in $\varphi 60$ are uniform in $\varphi 260$ on the circle. The target-substrate distance can be adjusted continuously from $40 \mathrm{~mm}$ to $80 \mathrm{~mm}$ by changing the height of the target. Change the deflection angle to adjust the eccentricity. With the increasing deflection angle from $0^{\circ}$ to $180^{\circ}$ the eccentricity is also increasing. Sputtering stainless steel materials for $15 \mathrm{~min}$ on the monocrystalline silicon substrate, then achieve the different samples. The sample size $45 \mathrm{~mm} \times 20 \mathrm{~mm}$, choose a measuring point every $5 \mathrm{~mm}$. There are 10 measuring points on every sample. And carried on the following test: (1) The target-substrate distance changing when the deflection angle and the rotational velocity are fixed; (2) The deflection angle is changing when the target-substrate distance and the rotational velocity are fixed; (3) The rotational velocity is changing when the deflection angle and the target-substrate distance are fixed. 


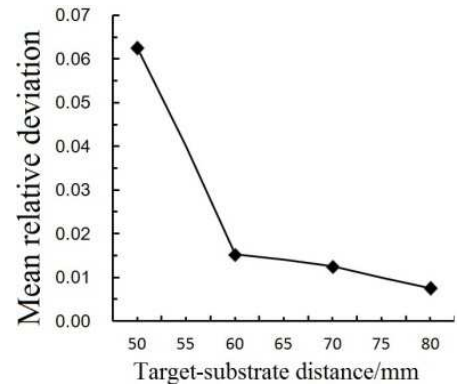

Fig. 11. Relation between mean relative deviation target-substrate distance

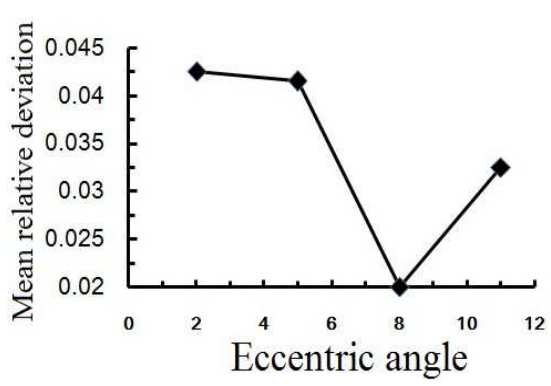

Fig. 12. Relation between mean relative deviation and eccentric angle

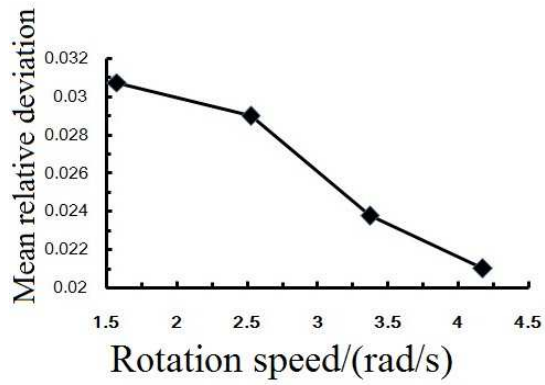

Fig. 13. Relation between mean relative and deviation and rotation speed

\section{Conclusions}

The paper presents a new system of magnetron sputtering with multi-workbench, establishes the corresponding film thickness distribution, calculates and analyses the film thickness when the substrate is in different positions and motions. Then achieving the conclusion: when the substrate is rotating with a fixed eccentricity in situ, the film thickness uniformity getting better because of the target-substrate distance increasing; when the substrate is rotating with a fixed target-substrate distance in situ, the film thickness uniformity getting better firstly and turning poor latter because of the target-substrate distance increasing; when the eccentricity ad the target-substrate distance are both fixed to increase the rotation-revolution ratio is good at achieving the uniform film in the lager area. Experimental data complies with the simulation conclusion in general.

\section{References}

[1] X.Q. Meng, D.Z. Ren, H.X. Guo, et al., Calculation of growth rate and thickness uniformity of the film deposited by cathode hollow magnetron sputtering, Journal of Wuhan University (Natural Science Edition), 41(1995) 351-356.

[2] F. Tatsuo, S. Fumihiko, N. Masahiko, Observations on the operation of a planar magnetron sputtering system by target erosion patterns, Thin Films, 153(1987) 373-381.

[3] L.G. Han, Researched of coating equipment and film uniformity on plane target by magnetron sputtering with multi-workbench, University Journal of Electronic Science and Technology of China, 20(2004) 24-25.

[4] D.L. Pan, M.S. Xiong, Y.D. Zhang, et al., Flim uniformity calculation of large caliber coating machine, High Power Laser and Particle Beams. 12(2000)277-280.

[5] M. Fang, X.Z Fan, J. B. Huang, Numerical simulation of a flat planetary fixture for physical vapor deposition, Chinese Journal of Computational Physics, 23(2006)738-742.

[6] X.D. Wang, D.C. Ba, S. W. Zhang, Y. C. Zhang, Vacuum Technology, Metallurgical Industry Press, Beijing, 2006. 